Chirurg 2021 · 92:665

https://doi.org/10.1007/s00104-021-01440-7

Angenommen: 18. Mai 2021

Online publiziert: 16. Juni 2021

(c) Springer Medizin Verlag GmbH, ein Teil von Springer Nature 2021

\section{Originalpublikation}

Seong Wook S, Keun Soo A, Sang Woo K et al (2021) Liver resection versus local ablation therapies for hepatocellular carcinoma within the Milan criteria: a systematic review and meta-analysis. Ann Surg 273(4):656-666. https:// doi.org/10.1097/SLA.0000000000004350.

Einleitung. Das hepatozelluläre Karzinom ist die sechsthäufigste Krebserkrankung weltweit. Aufgrund der sich verbessernden bildgebenden diagnostischen Verfahren und der engmaschigen Kontrolle von Patienten mit Risikofaktoren, gelingt die Diagnose eines hepatozellulären Karzinoms häufiger in der Frühphase. Die hier vorgestellte Arbeit beschäftigt sich mit der Frage, ob es einen Unterschied hinsichtlich des onkologischen Outcomes zwischen dem Ansatz der Leberresektion gegenüber dem der lokal ablativen Verfahren gibt. Die ausgewerteten Daten entstammen den Datenbanken von Medline, Embase und der Cochrane Library.

Methodik. Für diese Metaanalyse wurden insgesamt 7 randomisierte kontrollierte Studien (RCT) und 18 „gematchte“, nichtrandomisierte Studien (NRT) als relevante Studien eingeschlossen. Von den eingeschlossenen Patienten erhielten 2865 eine Leberteilresektion und 2764 wurden einem lokal ablativen Verfahren unterzogen. Als primärer Endpunkt betrachtete man das Gesamtüberleben. Als sekundäre Endpunkte definierte man das rezidivfreie Überleben (RFS) sowie das Rezidivmuster. Eingeschlossen wurden ausschließlich Patienten mit hepatozel-

C. Gebhardt $\cdot$ M. Ardelt ${ }^{1} \cdot$ U. Settmacher

${ }^{1}$ Klinik für Allgemein-, Viszeral- und Gefäßchirurgie, Jena, Deutschland

\title{
Therapie beim hepatozellulären Karzinom: Leberresektion vs. lokale Ablation
}

lulärem Karzinom innerhalb der MilanKriterien bei Diagnosestellung.

Ergebnisse. Im Vergleich des Gesamtüberlebens konnte in der Analyse der RCT kein signifikanter Unterschied festgestellt werden. Die NRT zeigten eine geringe Überlegenheit der Leberteilresektion gegenüber der lokal ablativen Therapie in Bezug auf das 3- und 5-Jahres-Überleben nach dem Random-effects-Modell. Sowohl in den randomisierten als auch in den nichtrandomisierten Studien konnte ein signifikant besseres rezidivfreies Überleben nach operativer Therapie festgestellt werden (Hazard Ratio: 3-JahresRFS 0,83 [RCT], 0,61 [NRT]; 5-JahresRFS 0,75 [RCT], 0,61 [NRT]). Weiterhin kommt es nach ablativer Therapie zu einem deutlich häufigeren Auftreten von Lokalrezidiven. Demgegenüber ergeben die Analysen eine geringere Zahl an postinterventionellen Komplikationen sowie einen insgesamt kürzeren Krankenhausaufenthalt im Vergleich zur Leberteilresektion.

\section{Kommentar}

Anhand der hier durchgeführten Metaanalyse konnte eine signifikante Überlegenheit der Leberteilresektion in Bezug auf das rezidivfreie Überleben und das Auftreten von Lokalrezidiven bei vergleichbarem Gesamtüberleben festgestellt werden. Es scheint, dass das bessere Überleben Folge des verminderten Auftretens von Lokalrezidiven ist. Hieraus ergibt sich, dass die Leberteilresektion aktuell eine bessere Therapie zur primären Tumorkontrolle im Vergleich zum interventionellen
Vorgehen darstellt. Aufgrund der geringeren Komplikationsrate und des weniger invasiven Vorgehens mit kürzerem Krankenhausaufenthalt stellt die lokal ablative Therapie dennoch eine gute Option, insbesondere als BridgingTherapie oder bei nichtoperablen Patienten, dar. Grenzen der hier vorliegenden Arbeit sind zum einen eine Heterogenität zwischen den analysierten Studien, insbesondere in Bezug auf das Followup nach der Therapie, und zum anderen die Verwendung von Sammeldaten ohne Metaanalyse der individuellen Patientendaten. Unberücksichtigt bleibt die sekundäre Therapieoption der Lebertransplantation.

\section{Korrespondenzadresse}

PD Dr. Dr. med. univ. M. Ardelt Klinik für Allgemein-, Viszeral- und Gefäßchirurgie

Am Klinikum 1, 07747 Jena, Deutschland Michael.Ardelt@med.uni-jena.de

Interessenkonflikt. C. Gebhardt, M. Ardelt und U. Settmacher geben an, dass kein Interessenkonflikt besteht. 